\title{
Je unklarer der Befund, desto eher braucht es eine diagnostische Eskalation
}

\author{
Hans-Peter Simmen
}

Universitätsspital Zürich

Serdar Koçer et al. [1] beschreiben die Leidensgeschichte eines 55-jährigen Mannes, der nach dem Aufstehen vom Sitz im Bus plötzlich Schmerzen in seiner rechten Hüfte verspürte. Verständlicherweise hat dieser Mann seinen Hüftbeschwerden zunächst keine besondere Beachtung geschenkt und auf Spontanheilung gehofft. Fünf Wochen später nahmen die Beschwerden derart zu, dass er hinken musste und daher seinen Arzt aufsuchte. Radiologisch wurde ein Ausriss des Trochanter minor gefunden. Noch immer wurde auf Spontanheilung gesetzt. Als im weiteren Verlauf unvermittelt noch Atemnot auftrat, ergab die Abklärung fast ein halbes Jahr nach Beginn der Beschwerden eine pathologische Fraktur durch ein metastasierendes Bronchialkarzinom.

Ein Ausriss des Trochanter minor ohne adäquates Trauma ist immer hochverdächtig auf eine pathologische Fraktur. Sowohl im konventionellen Bild als auch im zusätzlichen Computertomogramm war keine Ursache eruierbar. In dieser Situation wäre wohl ein MRI angebracht gewesen, weil Tumoren mit dieser Methode wesentlich besser erkennbar sind. Im «Retrospektoskop» ist es natürlich immer einfacher zu sagen, was man hätte tun sollen.

«Take-home message»: Wenn bei einem sehr ungewöhnlichen Befund konventionelle Bilder inklusive Com- putertomografie keine Erklärung bieten, sollte zügig eskaliert werden. Unter dem Strich sind ergänzende Abklärungen wohl immer noch billiger als Wochen und Monate bis zu Einleitung einer wirksamen Therapie zu verlieren. Ob die finale Prognose bei einem metastasierenden Bronchialkarzinom bei Entdeckung fünf Monate früher wirklich besser gewesen wäre, ist ungewiss. Es ist zu befürchten, dass die Metastase im Trochanter minor nicht die einzige ist. Immerhin wäre die Leidensgeschichte der Hüftbeschwerden kürzer und die Osteosynthese der Fraktur zeitgerechter gewesen.

\section{Bibliografie}

Koçer S, Grácio S, Molnar P, Baumgartner L: A Non-traumatic avulsion fracture of the lesser trochanter: other diagnosis not to be missed. Praxis. 2021;110:105-107.

Prof. em. Dr. Hans-Peter Simmen

Klinik für Traumatologie

Universitätsspital Zürich

8091 Zürich

hanspeter.simmen@usz.ch 